SCIENCE CHINA

Physics, Mechanics \& Astronomy

\title{
Chaotic ray dynamics enables photonics with broadband light
}

\author{
Hailin Wang*, and Jens Nöckel \\ Department of Physics, University of Oregon, Eugene, OR 97403, USA
}

Received October 25, 2017; accepted October 29, 2017; published online November 1, 2017

$\begin{array}{ll}\text { Citation: } & \text { H.Wang, and J. Nöckel, Chaotic ray dynamics enables photonics with broadband light, Sci. China-Phys. Mech. Astron. 61, 014231 (2018), doi: } \\ \text { 10.1007/s11433-017-9128-4 }\end{array}$

Propagation of sound waves through reflections along a curved surface was first theoretically analyzed by Lord Rayleigh to explain the whispering gallery waves in St. Paul's Cathedral in London. Similar whispering gallery sound waves are now a popular tourist attraction at the Echo Wall of the Temple of Heaven in Beijing. Whispering gallery phenomena also exist for light waves in dielectric spheres, disks, or toroids where total internal reflection allows the light to circulate with little attenuation and interfere with itself to form optical resonances or modes. These "whispering gallery modes" have found applications ranging from photonics, quantum physics, biosensing, to optical clocks $[1,2]$.

Essential properties of the whispering gallery modes, such as the caustics that characterize their field profile, can also be understood with a particle-like model, for which a particle bounces elastically along the surface of a billiard. For a circular billiard, the reflection process preserves the angular momentum of the particle [3]. Correspondingly, a whispering gallery light wave retains a constant effective angular momentum as it propagates inside the circular optical resonator, with only minor evanescent leakage due to the wave nature of light. In order to launch the whispering gallery light wave, the angular momentum of the input light wave has to match that of the light wave circulating inside the circular billiard. The same is also true if the emission or output from a whispering gallery mode is to be collected by an adjacent waveguide. This turns out to be extremely difficult to do for light waves that cover a broad frequency spectrum, such as optical pulses

*Correspondence author (email: hailin@uoregon.edu) with a duration of a few femtoseconds, since the broad spectrum corresponds to a large spread in the effective angular momentum.

This stumbling block is especially frustrating because the physics underlying total internal reflection is largely frequency-independent and is thus ideally suited for broadband applications. Overcoming the input-output coupling problem while maintaining the advantages of the whispering gallery effect is therefore a major step forward.

The classical billiard-ball model suggests a possible solution: the angular momentum of the bouncing particle is no longer conserved if the billiard deviates from a circular symmetry. With sufficient deformation, the trajectory of the particle can even become chaotic, meaning that it is nearly impossible to predict the exact trajectory of the particle. This is not necessarily a bad thing. Nöckel and Stone [3] proposed some years ago to harness these dynamical processes that do not conserve angular-momentum to make better optical devices. These ideas have since been exploited and extended in a number of experimental settings [4,5]. For example, directional laser emissions have been achieved in a whispering gallery semiconductor laser, which would otherwise feature an isotropic emission pattern [6]. Efficient excitation of whispering gallery light waves with a laser beam in free space has also been achieved, which would otherwise require evanescent waves from an optical waveguide [7].

In a recent experiment, Jiang and his collaborators [8] have taken advantage of dynamical processes in a deformed whispering gallery optical resonator to solve the problem of angular momentum matching. They have designed and fabricated a silica toroidal resonator that features the rightv amount of 


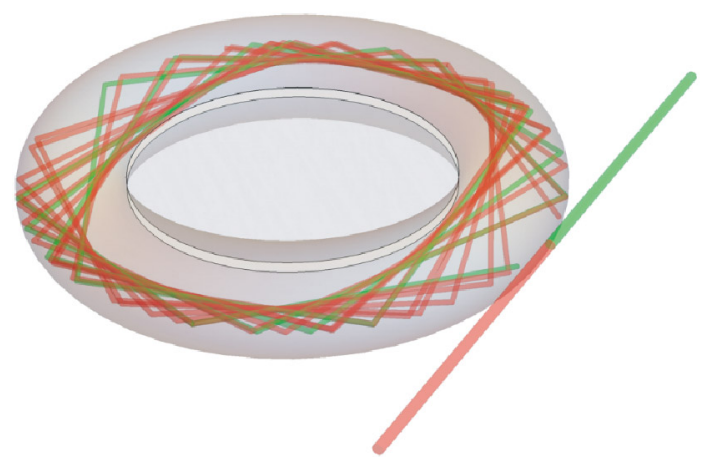

Figure 1 (Color online) Schematic illustrating angular momentum diffusion of both the input and the third harmonic light rays in a silica toroid. The third-harmonic light ray undergoes variations in the angular momentum, coupling out efficiently into the waveguide.

deformation (about 4\%). The deformation is sufficiently large to let broadband light traverse a large spread of angular momenta in a few picoseconds, but is not excessive so light can still circulate in the resonator for a long time.

As an example of an application, Jiang and his collaborators also used the silica resonator for third-harmonic generation. The third harmonic of the input laser light is generated through nonlinear optical processes in the toroid, which are enhanced by the high field strength achieved in a whispering gallery mode. In a circular toroid, light at the third harmonic cannot satisfy the angular momentum conservation required to return into the waveguide used for the input laser. By comparison, for a deformed toroid, the third-harmonic finds its way back through the chaotic trajectories and efficiently couples out the resonator, as illustrated schematically in Figure 1.

The experimental approach developed and demonstrated by Jiang and his collaborators breaks away from the conventional approach to satisfy momentum or angular momentum conservation in input-output optical coupling. This approach can enable new nanophotonic circuits and devices that involve broadband light.

1 K. J. Vahala, Nature 424, 839 (2003).

2 Y. F. Xiao, and Q. Gong, Sci. Bull. 61, 185 (2016).

3 J. U. Nöckel, and A. D. Stone, Nature 385, 45 (1997).

4 H. Cao, and J. Wiersig, Rev. Mod. Phys. 87, 61 (2015).

5 X. F. Jiang, C. L. Zou, L. Wang, Q. Gong, and Y. F. Xiao, Laser Photon. Rev. 10, 40 (2016).

6 C. Gmachl, F. Capasso, E. E. Narimanov, J. U. Nockel, A. Douglas Stone, J. Faist, D. L. Sivco, and A. Y. Cho, Science 280, 1556 (1998).

7 Y. S. Park, and H. Wang, Nat. Phys. 5, 489 (2009).

8 X. Jiang, L. Shao, S. X. Zhang, X. Yi, J. Wiersig, L. Wang, Q. Gong, M. Lončar, L. Yang, and Y. F. Xiao, Science 358, 344 (2017). 